\title{
The study of porous elastic plates by mesh-free methods
}

\author{
V. Sladek ${ }^{1}$, J. Sladek ${ }^{1}$, B. Musil ${ }^{2}$ \& L. Sator ${ }^{1}$ \\ ${ }^{1}$ Institute of Construction and Architecture, \\ Slovak Academy of Sciences, Slovakia \\ ${ }^{2}$ Department of Mechanics, Slovak Technical University, Slovakia
}

\begin{abstract}
Analytical formulae have been derived for the effective Young modulus and Poisson ratio of a homogeneous isotropic continuum used for replacing the porous linear elastic medium involving vacant elliptic pores. A strong formulation with the meshless approximation of field variables has been developed for a numerical solution to bending problems within three theories: Kirchhoff-Love theory, $1^{\text {st }}$ order and $3^{\text {rd }}$ order Shear Deformation Plate Theory. The influence of porosity on bending is studied numerically and presented for a circular plate.

Keywords: vacant elliptic pores, effective material coefficients, plate bending, thin and thick plates, mesh-free strong formulation, point interpolation method.
\end{abstract}

\section{Introduction}

Many advanced materials, like ceramics contain pores which lead the Young's modulus and strength to be low. Therefore a great attention is paid to prediction on elastic modulus of porous materials for a long time with broad scientific and technological importance. In general, it is difficult to analyse accurately the influence of pores on material coefficients, since the pores are distributed with random shape, size and orientation. It would be practically impossible to include defects of materials into numerical modelling of structures. Therefore the concept of replacement of defected (porous) materials by a homogeneous one with effective material coefficients seems to be very reasonable. Several empirical expressions to correlate porosity and Young's modulus have been reported in literature. Such relationships often have not a significant physical meaning. 
Intensive study has been based also on the theory of elasticity with embedding various defects into an elastic matrix continuum (see e.g. [1]).

In this paper, we shall study the influence of porosity on bending of elastic plates. The porosity is due to empty ("dry") pores of either circular or elliptical shape. The knowledge of the stress-deformation state in case of single pore embedded in an infinite isotropic and homogeneous linear elastic continuum is utilized for analytical derivation of the effective material coefficients in the representative volume element (RVE) whose boundaries are sufficiently far from the pore. Such effective material coefficients are applicable directly only for non-interacting pores, so for low levels of porosity. The interaction among the pores can be assessed via a correction factor. The random orientation of elliptic holes is considered in evaluation of effective material coefficients by angular averaging. For defects (pores) of arbitrary shape, one can apply the numerical analysis of the stress-deformation state in the RVE subjected to simple tension with assuming the periodic distribution of the RVE. Recall that in the case of high levels of porosity (dense packing of pores in the RVE), the numerical calculations fail because of inappropriate interactions of pores with fictitious boundaries of the RVE. Nevertheless, the numerical results for single pores are applicable also for higher densities of pores in combination with the correction factor.

Having known the effective material coefficients in the porous media, we studied the dependence of deflections, bending moments and shear forces in plates within the classical Kirchhoff-Love theory for bending of thin elastic plates as well as within the shear deformation plate theory valid also for thick plates. The strong formulation for solution of boundary value problems is employed with using meshless approximations for field variables. The high order derivatives in the governing equations are eliminated by using a decomposition technique.

\section{Effective elastic material coefficients}

Let us consider a linear elastic material with one pore under remote stress $\sigma_{i j}^{\infty}$. The average strains in the domain $\Omega$ of a sample are given as

$$
\left\langle\varepsilon_{i j}\right\rangle=\frac{1}{2 \Omega} \int_{\Omega}\left(u_{i, j}+u_{j, i}\right) d \Omega .
$$

In view of the Gauss divergence theorem, Eq. (1) can be rewritten as

$$
\begin{gathered}
\left\langle\varepsilon_{i j}\right\rangle=\varepsilon_{i j}^{0}+\Delta \varepsilon_{i j}, \quad \varepsilon_{i j}^{0}=\frac{1}{2 \Omega} \int_{\partial \Omega}\left(u_{i} n_{j}+u_{j} n_{i}\right) d \Omega, \\
\Delta \varepsilon_{i j}=\frac{1}{2 \Omega} \int_{\Gamma}\left(u_{i} n_{j}+u_{j} n_{i}\right) d \Gamma,
\end{gathered}
$$


where $\partial \Omega$ is the outer boundary of the domain $\Omega$, while $\Gamma$ is the boundary of the pore. Then, $\varepsilon_{i j}^{0}$ is the strain tensor in the sample without any pore, while $\Delta \varepsilon_{i j}$ is the disturbance strain due to the pore in the sample far from the pore. The same formula for $\Delta \varepsilon_{i j}$ has been employed also by Wang et al. [2]. Since the medium is linear elastic, both the strains $\varepsilon_{i j}^{0}$ and $\Delta \varepsilon_{i j}$ must be linear functions of applied stress

$$
\varepsilon_{i j}^{0}=M_{i j k l} \sigma_{k l}^{\infty}, \quad \Delta \varepsilon_{i j}=H_{i j k l} \sigma_{k l}^{\infty},
$$

where the tensor of compliance coefficients $M_{i j k l}$ is well known for homogeneous isotropic elastic media, while $H_{i j k l}$ should be determined. In view of Eqs. (2) and (3), we obtain

$$
\left\langle\varepsilon_{i j}\right\rangle=\bar{M}_{i j k l} \sigma_{k l}^{\infty}, \quad \bar{M}_{i j k l}=M_{i j k l}+H_{i j k l},
$$

with $\bar{M}_{i j k l}$ being the effective compliance tensor.

\subsection{Elliptical pore}

Let us consider a small elliptical hole in a finite size sample loaded on its boundary. The displacements in such a homogeneous and linear elastic sample with the hole can be assessed by the displacements in infinite elastic plane.

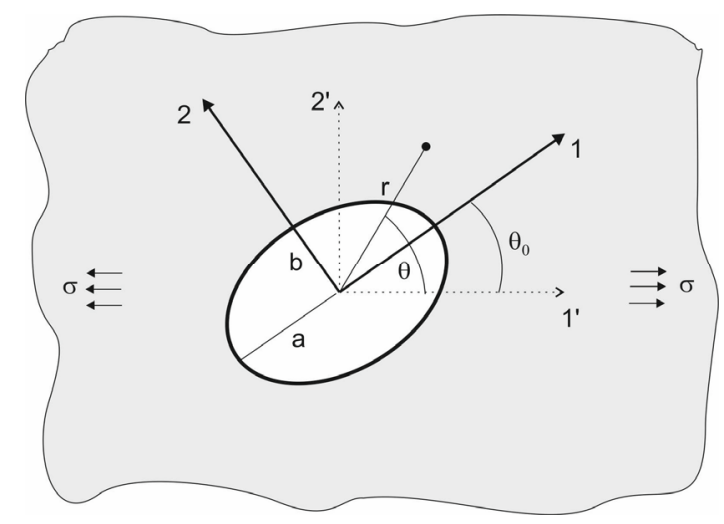

Figure 1: Elliptical hole in infinite plane with applied uniform stress loading in infinity. 
The deformation state in such a plane problem is usually solved via Airy stress function $U$, which can be found using functions of complex variable [3-5] and conform mapping of the plane with elliptical hole into the plane with unit circular hole. Without going into details, we present the displacements on the contour of the elliptic hole given as

$$
\begin{aligned}
& u_{1}(1, \theta)=\frac{\sigma R}{4} \frac{1+v}{E_{0}}\left\{\cos \theta\left[\Lambda(1-2 m)+\frac{1}{A}\left(1-2 m+2 m^{2}\right)\right]+\right. \\
& +\cos \left(\theta+2 \theta_{0}\right)\left[2(\Lambda+1)+\frac{2}{m}-2 \frac{1+m^{2}}{A m}\right]+ \\
& \left.+\cos \left(\theta-2 \theta_{0}\right) \frac{2}{A}+\cos 3 \theta \frac{m(m-2)}{A}+\cos \left(3 \theta+2 \theta_{0}\right) \frac{2}{A}\right\} \\
& u_{2}(1, \theta)=\frac{\sigma R}{4} \frac{1+v}{E_{0}}\left\{\sin \theta\left[\Lambda(1+2 m)+\frac{1}{A}\left(1+2 m+2 m^{2}\right)\right]+\right. \\
& +\sin \left(\theta+2 \theta_{0}\right)\left[-2(\Lambda+1)+\frac{2}{m}-2 \frac{1+m^{2}}{A m}\right]- \\
& \left.-\sin \left(\theta-2 \theta_{0}\right) \frac{2}{A}-\sin 3 \theta \frac{m(m+2)}{A}+\sin \left(3 \theta+2 \theta_{0}\right) \frac{2}{A}\right\} \text {, }
\end{aligned}
$$

where $R=(a+b) / 2,0 \leq m=(a-b) /(a+b)<1$.

In order to get the disturbance strains in the considered infinite plane, according to Eq. (2) we need to integrate along the elliptical hole. Performing such integrations, we obtained

$$
\left(\begin{array}{c}
\Delta \varepsilon_{11} \\
\Delta \varepsilon_{22} \\
\Delta \varepsilon_{12}
\end{array}\right)=\left(\begin{array}{lll}
H_{1111} & H_{1122} & 2 H_{1112} \\
H_{2211} & H_{2222} & 2 H_{2212} \\
H_{1211} & H_{1222} & 2 H_{1212}
\end{array}\right)\left(\begin{array}{c}
\sigma \cos ^{2} \theta_{0} \\
\sigma \sin ^{2} \theta_{0} \\
-\left(\sigma \sin 2 \theta_{0}\right) / 2
\end{array}\right)
$$

with $[H]=p \frac{1+v}{E_{0}}\left(\begin{array}{ccc}B_{1} & B_{2} & 0 \\ B_{3} & B_{4} & 0 \\ 0 & 0 & B_{5}\end{array}\right)$, where

$$
B_{1}:=\frac{1+\gamma}{8}\left[\Lambda(3-2 m)+3-m-m^{2}\right], B_{2}:=\frac{1+\gamma}{8}\left[-\Lambda(1+2 m)-\left(1+m+m^{2}\right)\right],
$$




$$
\begin{gathered}
B_{3}:=\frac{1+\gamma}{8 \gamma}\left[\Lambda(2 m-1)-1+m-m^{2}\right], B_{4}:=\frac{1+\gamma}{8 \gamma}\left[\Lambda(3+2 m)+3+m-m^{2}\right], \\
B_{5}:=\frac{1}{2}\left[1+\frac{1}{2}\left(\gamma+\frac{1}{\gamma}\right)\right](\Lambda+1),
\end{gathered}
$$

$p:=\frac{\pi a b}{\Omega}, \gamma:=\frac{b}{a}, \Lambda=3-4 \tilde{v}, \tilde{v}=\left\{\begin{array}{cc}v /(1+v), & \text { for plane stress problems } \\ v, & \text { otherwise. }\end{array}\right.$

Since $B_{2} \neq B_{3}$, the porous material with fixed orientation of the elliptic hole is not orthotropic. Let us consider the material with unspecified orientation of the elliptic hole. Then, we should consider angularly averaged values $\langle[H]\rangle_{\psi}$ instead of material coefficients $[H]$ obtained for a fixed orientation, with the angular averaging being defined as

$$
\left\langle H_{i j k l}\right\rangle_{\psi}:=\frac{1}{2 \pi} \int_{0}^{2 \pi} H_{i j k l}^{\prime \prime} d \psi, \quad H_{i j k l}^{\prime \prime}=\mathrm{O}_{i \alpha}^{T}(\psi) \mathrm{O}_{j \beta}^{T}(\psi) \mathrm{O}_{k \gamma}^{T}(\psi) \mathrm{O}_{l \delta}^{T}(\psi) H_{\alpha \beta \gamma \delta}
$$

After angular averaging, the effective medium is homogeneous isotropic and linear elastic continuum with the effective Young modulus and Poisson ratio being given by

$$
\bar{E}:=\frac{E_{0}}{1+p(1+v) A_{1}}, \quad \bar{v}:=\frac{v-p(1+v) A_{2}}{1+p(1+v) A_{1}} \text { for plane stress formulation, }
$$

while for plane strain formulation, we have

$$
\bar{E}:=\frac{E_{0}}{1+v} \frac{1+v+p\left(A_{1}-2 A_{2}\right)}{\left[1+p\left(A_{1}-A_{2}\right)\right]^{2}}, \quad \bar{v}:=\frac{v-p A_{2}}{1+p\left(A_{1}-A_{2}\right)} .
$$

In order to get the effective Young modulus for the case with interacting pores, it has been shown [2] that these can be found from those for the single pore by using certain correction factor as

$$
E^{e f f}=(1-p) \bar{E}
$$




\section{Shear deformation plate theory (SDPT) and Kirchhoff-Love theory (KLT)}

Let us consider a straight plate structure occupying the 3D domain

$$
V=\left\{\forall\left(x_{1}, x_{2}, x_{3}\right) \in \mathbb{R}^{3} ; \mathbf{x}=\left(x_{1}, x_{2}\right) \in \Omega, x_{3} \in[-h / 2, h / 2]\right\}=\Omega \times[-h / 2, h / 2]
$$

In order to unify the formulation for three theories, KLT, $1^{\text {st }}$ order SDPT (FSDPT) and $3^{\text {rd }}$ order SDPT (TSDPT), we can write the displacements as

$$
v_{i}\left(\mathbf{x}, x_{3}\right)=\delta_{i \alpha}\left\{u_{\alpha}(\mathbf{x})+\left[c_{1} \phi\left(x_{3}\right)-x_{3}\right] w_{, \alpha}(\mathbf{x})+c_{1} \phi\left(x_{3}\right) \varphi_{\alpha}(\mathbf{x})\right\}+\delta_{i 3} w(\mathbf{x})
$$

where $u_{\alpha}(\mathbf{x}), w(\mathbf{x})$ and $\varphi_{\alpha}(\mathbf{x})$ stand for in-plane displacement, deflection and rotation fields, respectively, while $\phi\left(x_{3}\right):=x_{3}-c_{2} \psi\left(x_{3}\right), \psi\left(x_{3}\right):=4\left(x_{3}\right)^{3} / 3 h^{2}$. The keys $c_{1}$ and $c_{2}$ switch among the considered theories according to the choices

$$
c_{1}=\left\{\begin{array}{l}
0, \mathrm{KLT} \\
1, \mathrm{SDPT}
\end{array}, \quad c_{2}=\left\{\begin{array}{ll}
0, & \text { FSDPT } \\
1, & \mathrm{TSDPT}
\end{array} .\right.\right.
$$

Making use the principle of virtual work, one can derive the governing equations

$$
\begin{gathered}
T_{\alpha \beta, \beta}=0 \\
\left(M_{\alpha \beta}-c_{1} \tilde{M}_{\alpha \beta}\right)_{, \alpha \beta}+c_{1} \tilde{T}_{3 \alpha, \alpha}=-q \\
c_{1}\left(\tilde{M}_{\alpha \beta, \beta}-\tilde{T}_{3 \alpha}\right)=0
\end{gathered}
$$

where $T_{\alpha \beta}(\mathbf{x})$ are the averaged in-plane stresses, $M_{\alpha \beta}(\mathbf{x}), S_{\alpha \beta}(\mathbf{x})$ are averaged stress couples, $T_{3 \beta}(\mathbf{x}), Q_{3 \beta}(\mathbf{x})$ are averaged transverse shear stresses and $\tilde{M}_{\alpha \beta}:=M_{\alpha \beta}-c_{2} S_{\alpha \beta}, \quad \tilde{T}_{3 \beta}:=T_{3 \beta}-c_{2} Q_{3 \beta}$. 
It is appropriate to use dimensionless formulation specified as follows

$$
\begin{aligned}
& x_{\alpha}^{*}=\frac{x_{\alpha}}{L}, x_{3}^{*}=\frac{x_{3}}{h}, u_{\alpha}^{*}=\frac{u_{\alpha}}{h}, \quad \varphi_{\alpha}^{*}=\varphi_{\alpha}, \quad w^{*}=\frac{w}{h}, \quad T_{\alpha \beta}^{*}(\mathbf{x})=\frac{L^{2}}{D} T_{\alpha \beta}(\mathbf{x}), \\
& T_{3 \alpha}^{*}=\frac{h L}{D} T_{3 \alpha}, Q_{3 \alpha}^{*}=\frac{h L}{D} Q_{3 \alpha}, M_{\alpha \beta}^{*}=\frac{L^{2}}{D h} M_{\alpha \beta}, S_{\alpha \beta}^{*}=\frac{L^{2}}{D h} S_{\alpha \beta}, q^{*}:=\frac{L^{4}}{D h} q
\end{aligned}
$$

with $D:=\frac{E h^{3}}{12\left(1-v^{2}\right)}$ being the bending stiffness. Then, the governing equations become

$$
\begin{aligned}
T_{\alpha \beta, \beta}^{*}=0, \quad\left(M_{\alpha \beta}^{*}-c_{1} \tilde{M}_{\alpha \beta}^{*}\right)_{, \alpha \beta}+c_{1}\left(\frac{L}{h_{0}}\right)^{2} \tilde{T}_{3 \alpha, \alpha}^{*}=-q^{*}, \\
c_{1}\left[\tilde{M}_{\alpha \beta, \beta}^{*}-\left(\frac{L}{h_{0}}\right)^{2} \tilde{T}_{3 \alpha}^{*}\right]=0 .
\end{aligned}
$$

Note that there is no coupling between bending and the in-plane deformations provided that the Young modulus is not graded along the plate thickness. In what follows, we shall be interested only in the bending problems and rewrite the governing equations in terms of the primary fields $w(\mathbf{x})$ and $\varphi_{\alpha}(\mathbf{x})$.

\section{KLT:}

$$
\nabla^{2} m^{*}=q^{*}, \quad \nabla^{2} w^{*}-m^{*}=0
$$

FSDPT:

$$
\begin{gathered}
6(1-v) k\left(\frac{L}{h}\right)^{2}\left(w_{, \alpha \alpha}^{*}+\frac{L}{h} \varphi_{\alpha, \alpha}^{*}\right)=-q^{*} \\
f_{\alpha}^{*}+\frac{1+v}{1-v} \varphi_{\beta, \beta \alpha}^{*}-12 k\left(\frac{L}{h}\right)\left(w_{, \alpha}^{*}+\frac{L}{h} \varphi_{\alpha}^{*}\right)=0, \quad \nabla^{2} \varphi_{\alpha}^{*}-f_{\alpha}^{*}=0
\end{gathered}
$$

TSDPT:

$$
4 \frac{L}{h}(1+v) f_{\alpha, \alpha}^{*}-\nabla^{2} m^{*}=-q^{*}
$$




$$
\begin{gathered}
17\left[(1-v) f_{\alpha}^{*}+(1+v) \varphi_{\gamma, \gamma \alpha}^{*}\right]-13 \frac{h}{L} m_{, \alpha}^{*}-168(1-v) \frac{L}{h}\left(w_{, \alpha}^{*}+\frac{L}{h} \varphi_{\alpha}^{*}\right)=0 \\
\nabla^{2} w^{*}-m^{*}=0, \quad \nabla^{2} \varphi_{\alpha}^{*}-f_{\alpha}^{*}=0
\end{gathered}
$$

The new field variables $m^{*}$ and $f_{\alpha}^{*}$ have been introduced in order to decrease the order of the derivatives of the primary field variables [6]. Now, the maximum order of the derivatives is equal to 2 instead of 4 occurring in the formulation without decomposition. This is important from the point of view of accuracy of derivatives of approximated field variables, especially in case of strong formulations.

In numerical solution, we apply the strong formulation with meshless approximation for field variables by Point Interpolation Method (PIM) using the radial bases functions (multiquadrics) and polynomials [6, 7]. The governing equations are collocated at interior nodes while the boundary conditions at nodes on the boundary contour.

\section{Numerical examples}

Since the porous medium is assumed to be replaced by a homogeneous, isotropic and linear elastic continuum with an effective Young modulus and Poisson ratio, the plate bending problems for such porous plates looks rather simply. For illustration, we present numerical results for circular plate with a central circular hole, for which the exact solution is also available within the KLT [6]. We present the numerical results for the parametric study of the deflection, slope, bending moment and generalized shear force in thin as well as thick plates for several values of the porosity $p \in[0,0.6]$. The uniform transversal loading is assumed

$q=D_{0} h /\left(r_{1}\right)^{4}$ and keeping constant for each calculation with different values of the porosity. The inner and outer radii are also kept constant, $r_{1}=1$ and $r_{0}=0.1 r_{1}$ in dimensionless coordinates and both the edges are clamped. Then, the dimensionless loading $q^{*}=D_{0} / D$ is dependent on the porosity. Because of the angular symmetry, the nodal points are distributed only along the radius of the plate and the presented results have been obtained by using a uniform distribution of 121 nodes. Good convergence of accuracy is achieved with increasing the amount of nodes and the $L_{2}$-norm error for deflections is less than $6 \times 10^{-3} \%$ when 121 nodes is employed.

Fig. 2 shows the dependence of the effective Young modulus and Poisson ratio on the porosity with considering two shapes of pores. The influence of the shape of pores on $E_{\text {eff }}$ is negligible, while it is significant on the effective Poisson ratio. 

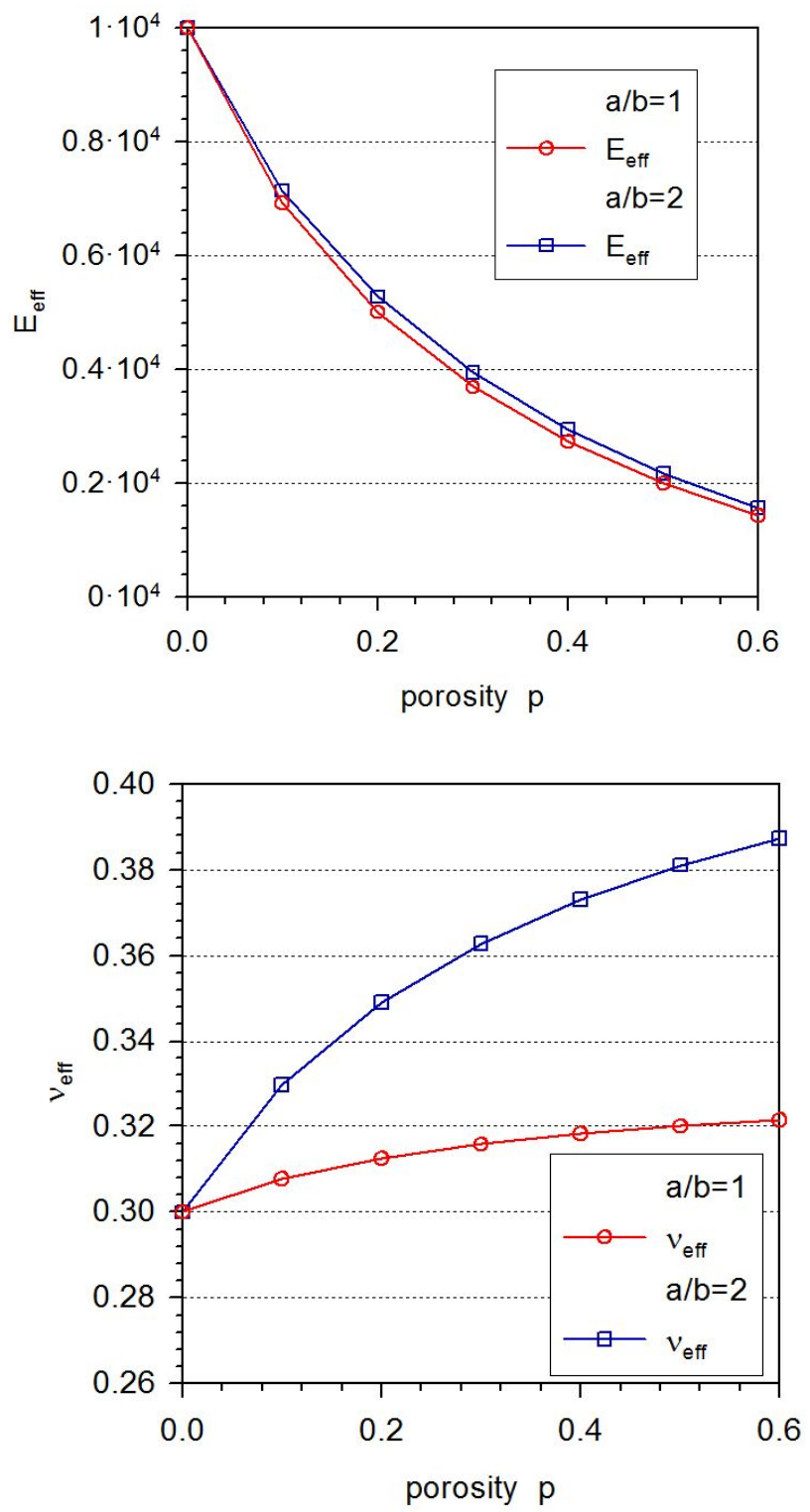

Figure 2: $\quad$ The effective Young modulus and Poisson ratio vs porosity for circular pores $(a / b=1)$ and elliptic pores $(a / b=2)$.

From Fig. 3, we can see a strong influence of the porosity on the investigated quantities. 
72 Boundary Elements and Other Mesh Reduction Methods XXXVII

$\frac{0}{\frac{0}{\pi}}$
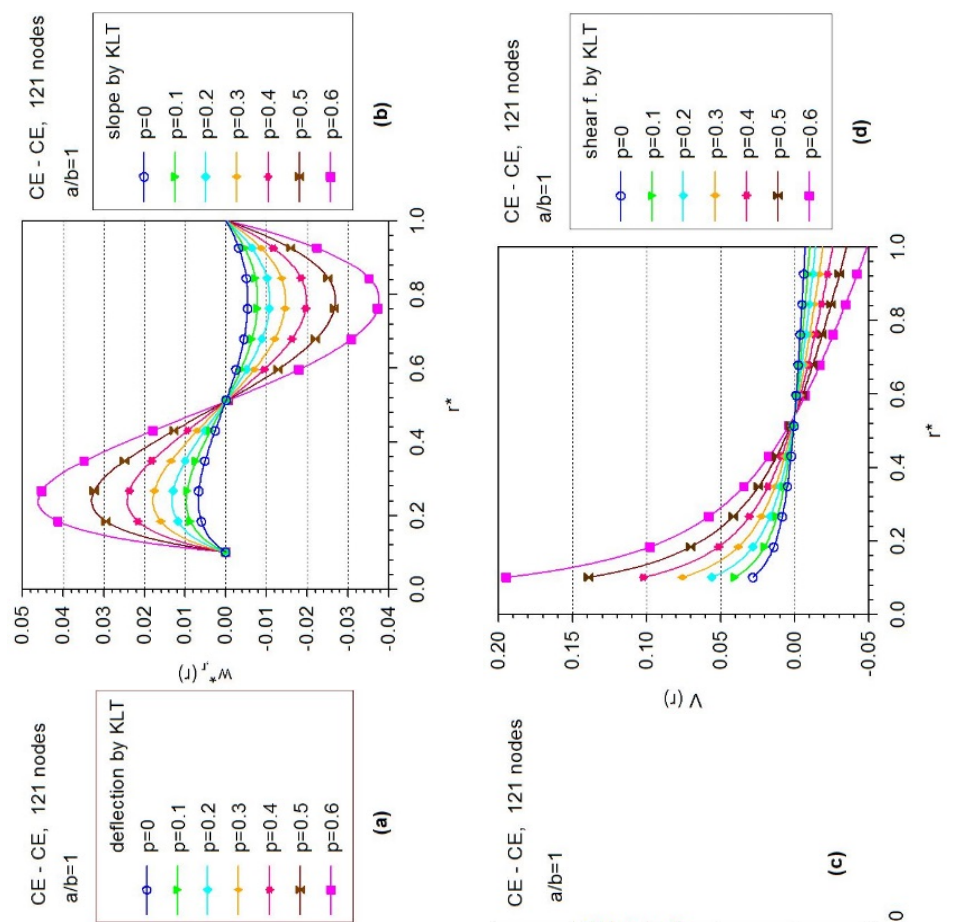

(ג) $\wedge$

ชั.

节

ర్ల

근

过

ซิ
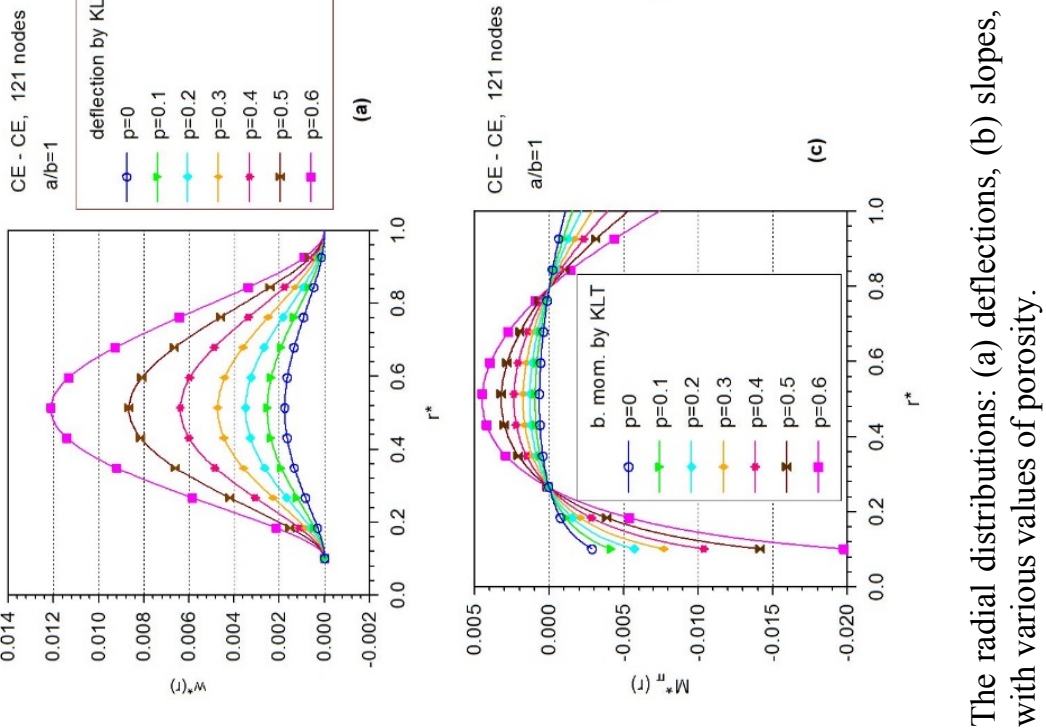

$\underset{0}{\ddot{0}}$ 
The dimensionless numerical results shown in Fig. 3 are obtained by the KLT and they do not depend on the ratio $r_{1} / h$. Having used $r_{1}=50 h$, the numerical results by the FSDPT as well as by the TSDPT are practically the same as those by the KLT. The differences between the KLT and SDPT results for various values of the ratio $r_{1} / h$ can be seen from Fig. 4 .

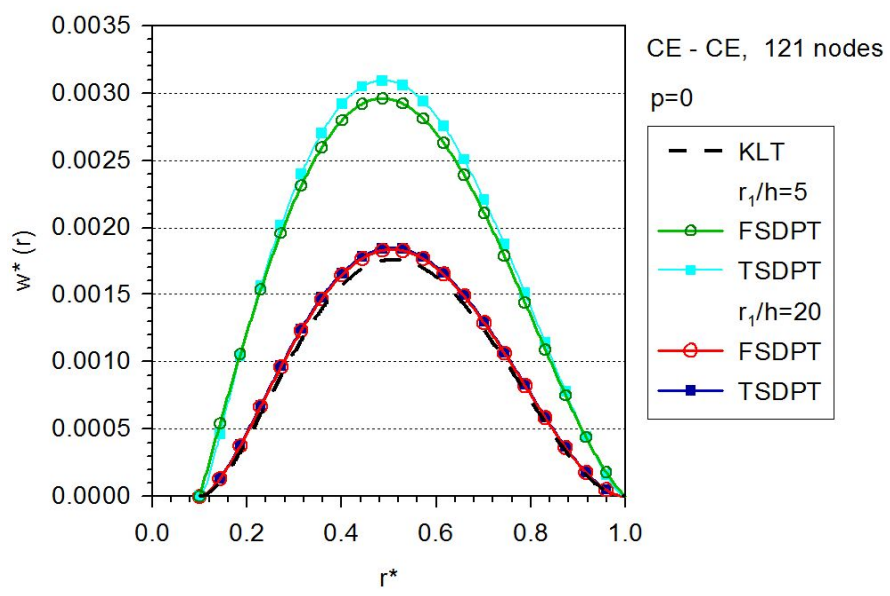

Figure 4: Comparison of numerically computed deflections by the KLT and SDPT for two values of the ratio $r_{1} / h$ in plate without porosity.

The influence of the shape of pores on deflections can be seen from Fig. 5.



Figure 5: Comparison of deflections in porous plates with two shapes of pores. 
It can be seen from Fig. 6 that the dependence of the maximal deflections on the porosity is nonlinear. The bending of the porous plate is affected by the porosity via both the Young modulus and the Poisson number. Furthermore, it is seen that the dependence is more expressive for thick plate. For thin plate $\left(r_{1}=50 h\right)$ all three theories give almost the same results, for the medium thickness $\left(r_{1}=10 h\right)$ there is coincidence only between the FSDPT and the TSDPT, and finally for the thick plate $\left(r_{1}=5 h\right)$ a deviation can be seen even between the FSDPT and the TSDPT results. The differences between the results by various theories are increasing with increasing the porosity.

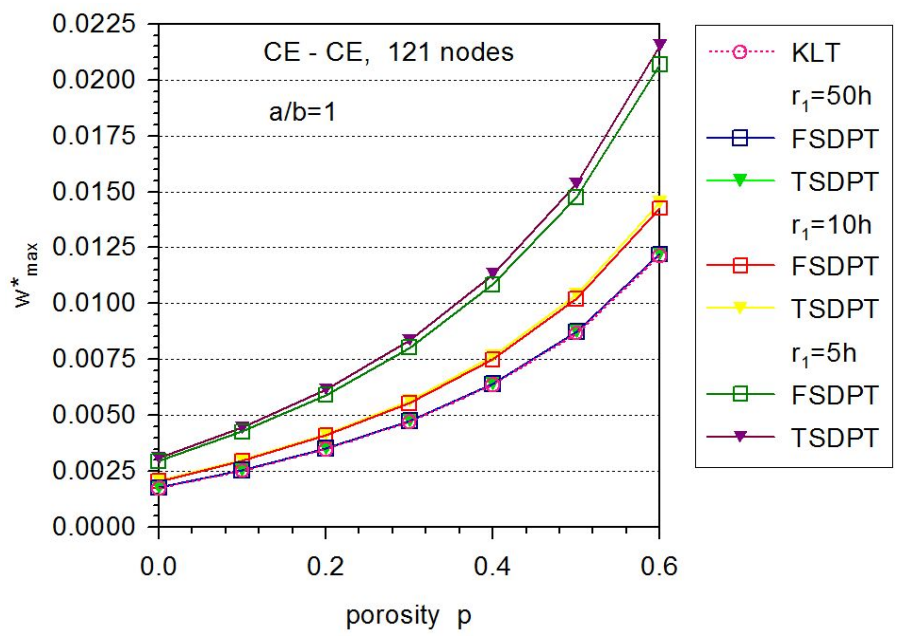

Figure 6: Dependence of maximal deflections on the porosity.

\section{Conclusions}

The analytical formulae have been derived for the dependence of Young's modulus and Poisson's number on the porosity for the effective homogeneous linear elastic continuum used for replacing the porous medium involving randomly oriented elliptic empty pores. The shape of pores has more significant influence on the effective Poisson ratio than on the effective Young modulus. The influence of porosity on bending of elastic plates has been studied in three theories: KLT, FSDPT and TSDPT followed by a discussion of the results.

\section{Acknowledgement}

This work was partially supported by the Slovak Research and Development Agency under the contract No. APVV-0032-10. 


\section{References}

[1] Miled, K., Sab, K., Le Roy, R., Effective elastic properties of porous materials: Homogenization schemes vs experimental data. Mech. Res. Comm. 38, pp. 131-135, 2011.

[2] Wang, F., Gou, W., Zheng, X., Lu, M., Effective elastic moduli of ceramics with pores. J. Mater. Sci. Technol. 14, pp. 286-288, 1998.

[3] Sedov, L.I., Mechanics of Continuous Media, Vol. 2, World Scientific Publ. Co.: Singapore, 1997.

[4] Lurie, A.I., Theory of Elasticity, Springer: Berlin, 2005.

[5] Muskhelishvili, N.I., Some Basic Problems of the Mathematical Theory of Elasticity, P. Noordhoff: Groningen, 1953.

[6] Sladek, V., Sladek, J., Sator, L., Physical decomposition of thin plate bending problems and their solution by mesh-free methods. Engineering Analysis with Boundary Elements 37, pp. 348-365, 2013.

[7] Liu, G.R., Mesh free methods, moving beyond the finite element method. CRC Press: Boca Raton, 2003. 